\title{
Improvements in Live Cell Analysis of G Protein Coupled Receptors using Second Generation BD Calcium Assay Kits
}

\author{
Xiao Li*, Isabel Llorente and Mike Brasch
}

Bioimaging Systems, BD Biosciences, Rockville, Maryland, USA

\begin{abstract}
BD}^{\mathrm{TM}}$ Calcium Assay Kits are designed for cell-based calcium mobilization high-throughput screening assays. The kits use a proprietary formulation including a non-fluorescent calcium indicator that becomes activated inside the cell and shows increased fluorescence upon calcium binding. The formulation includes a signal-enhancing reagent to maximize the signal over background in a homogeneous, no-wash assay format, based on a technology developed at BD. We have compared the next generation BD calcium assay kit product family to previous versions of the formulation, and to other commercially available homogeneous calcium assay kits. The improvements have enabled better performance on the cell lines and receptors that we have tested in all plate formats including 1536.
\end{abstract}

\section{INTRODUCTION}

GPCRs are known to play a crucial role in the development and progression of major diseases such as cardiovascular, respiratory, gastrointestinal, neurological, psychiatric, metabolic and endocrinological disorders [1]. Fluorescent calcium indicators, such as Fluo-3 and Fluo-4, have been widely used in the high-throughput screening field for GPCRs and other targets that result in calcium mobilization [2]. The protocols using Fluo-3 and Fluo-4 typically require time consuming washing steps before and after cells are loaded with fluorescent dyes. As the result of washing, cells are often lost from the microplates, resulting in well to well variation and decreased data quality. Harsh treatment of cells by washing can also diminish the cellular response to ligands.

Commercially available no-wash calcium assay formulations were developed by several companies to increase screening throughput and improve data quality of calcium mobilization assays [3]. An evaluation of no-wash calcium assay formulations using human native rhabdomyosarcoma cells expressing endogenous urotensin-II receptor was conducted by Xin et al. at Johnson \& Johnson, and demonstrated that the $\mathrm{BD}^{\mathrm{TM}}$ Calcium Assay Kit delivered the best data quality by providing higher signal to background and eliminating an addition artifact resulting from compound addition when other formulations were used [4].

After cells are loaded with calcium indicators, organicanion transporters located in the cell membrane can actively extrude the dyes back into extra-cellular solution. These transporters can be particularly active in certain cell types such as $\mathrm{CHO}$ and HeLa. In these cases, probenecid, a standard inhibitor of nonspecific anion transport, is often included in the dye-loading solution to reduce leakage [5]. The necessity to prepare a probenecid stock solution adds yet one more step in the screening campaign. Although in general probenecid does not alter free calcium concentrations in the

*Address correspondence to this author at the Bioimaging Systems, BD Biosciences, Rockville, Maryland, USA; E-mail: xiao_li@bd.com cytosol, it does have an apparent toxic effect on some cells after prolonged incubation. In addition, probenecid was also shown to change the calcium flux kinetics of a cyclic nucleotide gated ion channel and decrease the channel's response to agonists of Gs-couple receptors in the $\mathrm{BD}^{\mathrm{TM}}$ ACTOne GPCR assay [6]. To mitigate these negative effects of probenecid, novel calcium dye formulations were developed (BD ${ }^{\mathrm{TM}} \mathrm{PBX}$ Calcium Assay Kit). Probenecid titration experiments showed that the PBX kit was able to detect intracellular calcium flux in $\mathrm{CHO}$ cells in the absence of probenecid.

In this report we describe a second generation BD calcium assay kit. Comparison of the first and second generation formulations on various cell lines and receptors in 96, 384 and 1536-well format, showed the new formulation provides a higher signal to background and an increased robustness in GPCR screening campaigns.

\section{MATERIALS AND METHODS}

\section{Cell Culture}

Cell lines were purchased from ATCC (Manassas, VA). CHO-K1 cells were maintained in F-12 nutrient mixture with $10 \% \mathrm{FBS}$ and penicillin/streptomycin (Invitrogen, Carlsbad, $\mathrm{CA})$. CHO-K1 cells stably transfected with rat M1 muscarinic acetylcholine receptor (CHO-M1) were maintained in F-12 nutrient mixture with $10 \%$ FBS and $100 \mu \mathrm{g} / \mathrm{ml} \mathrm{G} 418$ (Invitrogen, Carlsbad, CA). CHO cells stably expressing rat muscarinic receptor 1 (CHO-M1) were maintained in F-12 nutrient mixture with $10 \%$ FBS and $250 \mu \mathrm{g} / \mathrm{ml} \mathrm{G} 418$. HEK 293 cells were grown in Dulbecco's Minimal Essential Media (DMEM) with 10\% FBS and penicillin/streptomycin (Invitrogen, Carlsbad, CA).

\section{Regent Preparation for Calcium Flux Assays}

Preparation of dye-loading solutions of $\mathrm{BD}^{\mathrm{TM}}$ calcium assay kits (BD Biosciences, Rockville, MD. Table 1) were performed as described in the manual and illustrated in a flow chart (Fig. 1). Calcium 3 kit was purchased from Molecular Devices (Sunnyvale, CA), and prepared in HBSS buffer (Invitrogen, Carlsbad) containing $20 \mathrm{mM}$ HEPES ( $\mathrm{pH}$ 7.4) according to the product insert sheet. Probenecid 
Table 1. Summary of BD Calcium Assay Kits

\begin{tabular}{|c|c|c|}
\hline & Regular Applications & Probenecid Sensitive Applications \\
\hline \hline First generation & $\mathrm{BD}^{\mathrm{TM}}$ Calcium Assay Kit & $\mathrm{BD}^{\mathrm{TM}} \mathrm{PBX}$ Calcium Assay Kit \\
\hline Second generation & $\mathrm{BD}^{\mathrm{TM}}$ High Performance Calcium Assay Kit & $\mathrm{BD}^{\mathrm{TM}} \mathrm{PBX}_{\text {High Performance Calcium Assay Kit }}$ \\
\hline
\end{tabular}

(Sigma, St Louis, MO) was included in dye-loading solutions in some experiments as indicated. Agonists ATP and carbachol (Sigma, St Louis, MO) were prepared in HBSS/HEPES solution at $5 \mathrm{X}$ final concentrations for experiments on FlexStation and FLIPR 3 (Molecular Devices, Sunnyvale, CA), and at $6 \mathrm{X}$ of the final concentrations for experiments on FDSS 7000 (Hamamatsu, Japan).

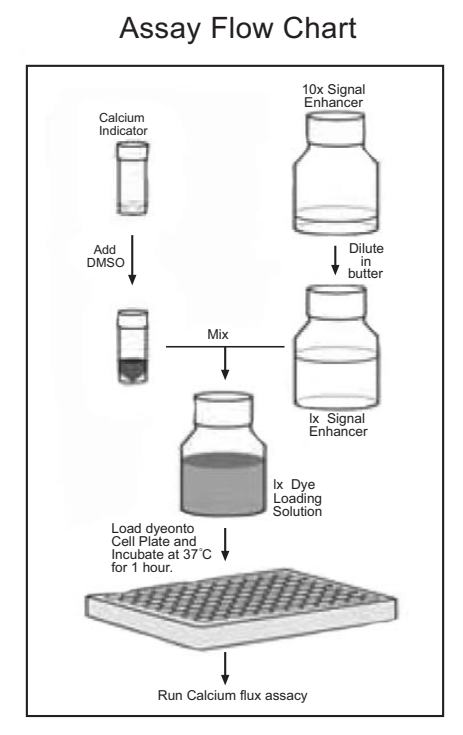

Fig. (1). Flow chart of dye-loading solution preparation.

\section{Calcium Flux Assays}

Experiments in 96-well plates. CHO cells and HEK293 cells were plated in $100 \mu \mathrm{l}$ culture medium on 96-well Biocoat poly-D lysine coated plates (BD Biosciences, Bedford, MA) at 50,000 cells per well. The next day, $100 \mu \mathrm{l} /$ well dyeloading solution was added to each well. After 1 hour of incubation at $37{ }^{\circ} \mathrm{C}$, calcium flux assays were performed with on-line compound addition in a FlexStation (Molecular Devices), with simultaneous data collection. Dose response curves were generated by SoftMax ${ }^{\circledR P r o ~(M o l e c u l a r ~ D e-~}$ vices).

Experiments in 384-well plates. CHO-K1, CHO-M1 cells and HEK293 cells were plated overnight in $25 \mu$ culture medium on 384-well Biocoat poly-D lysine coated plates (BD Biosciences, Bedford, MA) at 10,000 cells per well. The next day, cells were loaded with $25 \mu 1$ /well dye-loading solution at $37^{\circ} \mathrm{C}$ for 1 hour. Calcium flux assays were performed with on-line compound addition by either a FlexStation or a FLIPR 3 (Molecular Devices), with concurrent data collection. Dose response curves were generated by SoftMax ${ }^{\circledR}$ Pro (Molecular Devices). For antagonist assay, CHOM1 cells in a 384-well plate were incubated with pirenzepine
(Sigma, St Louis, MO) solution for 5 minutes before the ligand (40 $\mathrm{nM}$ carbachol) was added by a FlexStation.

Experiments in 1536-well plates. The experimental protocol was modified based on Liu et al. at NIH [7]. CHO-M1 cells were seeded overnight in $3 \mu$ l culture medium on 1536well black wall clear bottom plates (Greiner) at 2000 cells per well. The next day, cells were loaded with $2.5 \mu \mathrm{l} /$ well dye-loading solution at $37{ }^{\circ} \mathrm{C}$ for 1 hour. A FDSS 7000 (Hamamatsu, Japan) was used for calcium mobilization experiments, and $1 \mu$ lagonist or buffer was added to each well by the instrument and data were recorded simultaneously. Dose response curves were generated by Prism (Graphpad, San Diego, CA).

\section{RESULT}

\section{Comparison of First and Second Generation of $\mathbf{B D}^{\mathrm{TM}}$ Calcium Assay Kits}

To assess the performance of the BD calcium assay kits, the endogenous P2Y receptors in HEK293 [8] and CHO-K1 [9] cells were compared side by side using identical assay conditions. To maintain a true no-wash assay, culture medium was not removed before dye-loading. HEK 293 cells in general do not require probenecid for dye-loading, and therefore probenecid was not included in the dye-loading solution (Fig. 2A). However, probenecid was added to prevent dye leakage when $\mathrm{CHO}$ cells were assayed (Fig. 2B). The fluorescent signal change at different concentrations of the ligand ATP was calculated and plotted. As shown in Fig. (2), the second generation kit $\left(\mathrm{BD}^{\mathrm{TM}}\right.$ High Performance Calcium Assay Kit) exhibited improved signal to background in both HEK293 and CHO cells at the highest ATP concentrations, while the EC50 values remained similar.

Antagonsit assay was also conducted using both generations of calcium assay kits. Pirenzepine, a known muscarinic receptor inhibitor, was incubated with $\mathrm{CHO}$ cells overexpressing rat muscarinic receptor M1 for 5 minutes before the cells were applied with carbachol at EC80 value (40 nM in this case). As shown in Fig. (3), the assay using the second generation calcium assay kit delivered higher signal to background than the assay using the first generation kit. The IC 50 value remained similar between the two assays.

Assay miniaturization increases the speed and efficiency of screening and lead discovery. However, miniaturization of cell-based assays to a 1536-well format is challenging, due in part to difficulties in cell washing and obtaining appropriate plating densities. The impact of cell washing can be reduced by employing homogeneous true no-wash assay formats. We therefore evaluated the performance of both BD calcium formulations on $\mathrm{CHO}$ cells stably expressing the rat muscarinic receptor M1 (CHO-M1) in a 1536-well format using an FDSS 7000. Similar to the results obtained in 96 and 384-well formats above, the $\mathrm{BD}^{\mathrm{TM}}$ High Performance 
A ATP Dose Response Curves (HEK293)

B ATP Dose Response Curves (CHOK1)

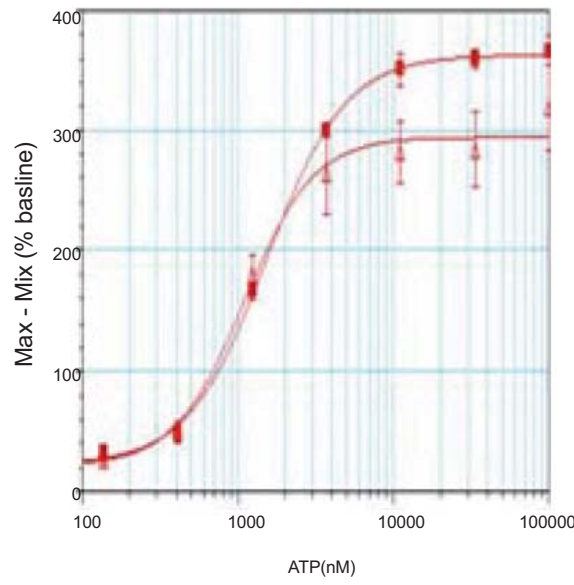

- BD ${ }^{\mathrm{TM}}$ High Performance Kit

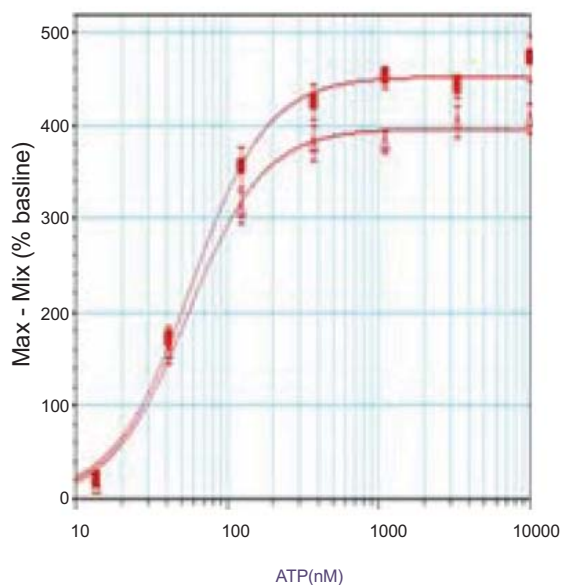

$\triangle$ BD $^{\text {TM }}$ Calcium Assay Kit

$\mathrm{n}=4$

Fig. (2). Response of endogenous P2Y receptor to ATP.

A. Dose response curves of ATP in HEK 293 cells in the absence of probenecid in 384-well format. Signal to background are 3.9 and 4.6 , EC50 values are $1.1 \mu \mathrm{M}$ and $1.5 \mu \mathrm{M}$ using the first and second generation kits, respectively.

B. Dose response curve of ATP in CHO-K1 cells in the presence of $2.5 \mathrm{mM}$ probenecid in 96 -well format. Signal to background are 5.0 and 5.5, EC50 values are $67 \mathrm{nM}$ and $58 \mathrm{nM}$ using the first and second generation kits, respectively.

Calcium Assay Kit provided higher response to carbachol, an agonist of muscarinic receptor, than the first generation kit (Fig. 4A). Both kits delivered robust signals, and there was no significant difference in EC50 and Z' values [10] (Fig. 4B).

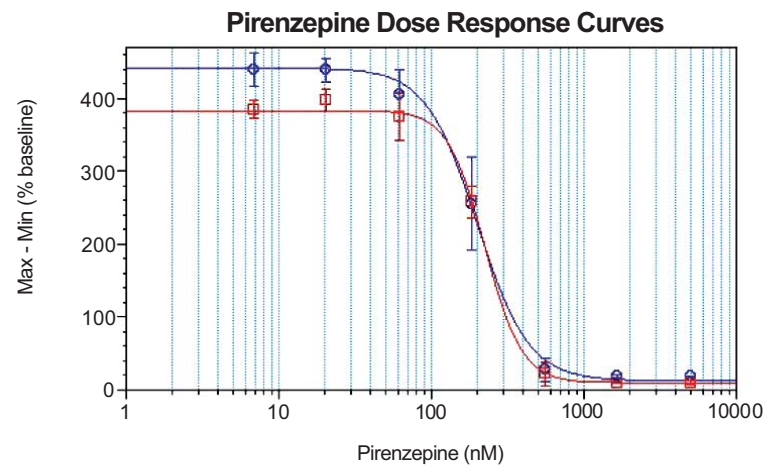

Fig. (3). Response of muscarinic receptor to its antagonist, pirenzepine.

Dose response curves of pirenzepine in CHO-M1 cells in the presence of $40 \mathrm{nM}$ carbachol at 384-well format. Signal to background are 4.8 and 5.4, IC50 values are $224 \mathrm{nM}$ and $200 \mathrm{nM}$ using the first and second generation kits, respectively.

$\bigcirc \mathrm{BD}^{\mathrm{TM}}$ High Performance Calcium Assay Kit (second generation).

$\square \mathrm{BD}^{\mathrm{TM}}$ Calcium Assay Kit (first generation).

\section{Probenicid-Free Screening of CHO Cells with the $\mathrm{BD}^{\mathrm{TM}}$ PBX Calcium Assay Kit}

Because of high organic anion transporter activity in $\mathrm{CHO}$ cells, fluorescent calcium dyes are poorly retained in the absence probenecid. In a standard protocol for $\mathrm{CHO}$ cell calcium flux assays using Fluo-3, Fluo-4 and other commercial kits, $2.5 \mathrm{mM}$ probenecid is required to achieve the optimal signal. Moreover, when $\mathrm{CHO}$ cells are transfected with GPCR genes, overexpression of the GPCR can have a negative impact on the host cells' ability to tolerate prolonged incubation with probenecid. The $\mathrm{BD}^{\mathrm{TM}} \mathrm{PBX}$ Calcium Assay Kit was specially designed for assays that are sensitive to probenecid, and the special formulation of the PBX kit helps increase dye retention in cells. To assess the performance, a probenecid titration assay ( 0 to $2 \mathrm{mM}$ probenecid) was performed and measured on a FlexStation using the first generation BD calcium assay kit, BD PBX calcium assay kit and a Calcium 3 kit purchased from Molecular Devices. The data indicates that probenecid is not required for dye-loading $\mathrm{CHO}$ cells if the $\mathrm{BD}^{\mathrm{TM}} \mathrm{PBX}$ Calcium Assay Kit is used (Fig. 5A) while $0.5 \mathrm{mM}$ probenecid is sufficient for the $\mathrm{BD}^{\mathrm{TM}}$ Calcium Assay Kit (Fig. 5B). In contrast, the Calcium 3 kit requires at least $2 \mathrm{mM}$ probenecid to exhibit a response (Fig. 5D).

The performance of first generation BD kits and the Calcium 3 kit in the absence of probenecid was also compared side by side using a FLIPR 3. Endogenous P2Y receptor in CHO cells (Fig. 6) was used as an example. In CHO cells, the $\mathrm{BD}^{\mathrm{TM}}$ PBX Calcium Assay Kit was observed to generate 18,000 RFU of response to ATP and BD ${ }^{\mathrm{TM}}$ Calcium Assay Kit had 3000 RFU of response, while the Calcium 3 kit failed to deliver a positive signal. High fluorescent background in wells loaded with Calcium 3 kit indicated that the fluorescent calcium dyes were pumped out of cells in the absence of probenecid. When the agonist was added to the wells after dye-loading, the fluorescent molecules in extracellular environment were diluted and therefore a significant drop of fluorescent signal was observed. Because of poor dye-retention in cells, no fluorescent calcium flux signal could be detected by Calcium 3 kit. 
A Carbachol Response on $\mathrm{CH}-\mathrm{M} 1$ Cells

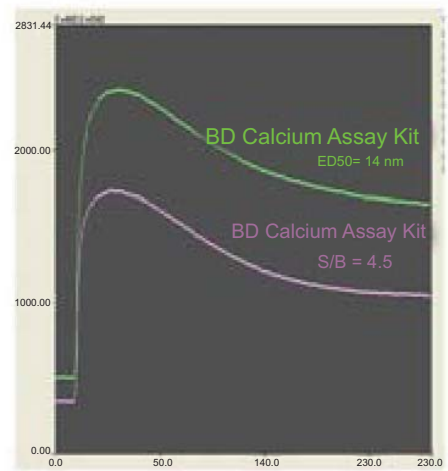

$B$

Carbachol Dose Response Curves

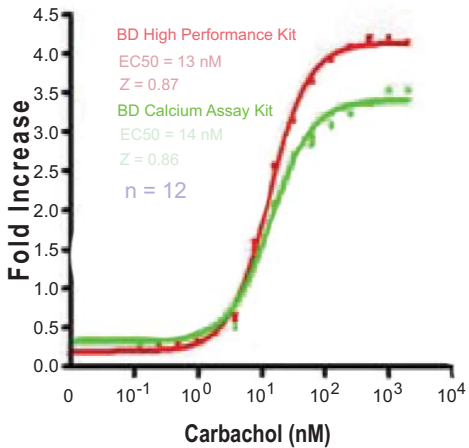

Fig. (4). Response of muscarinic receptor to carbachol in the presence of $2.5 \mathrm{mM}$ probenecid in 1536 -well format.

A. CHO-M1 cells treated with $1 \mu \mathrm{M}$ carbachol.

B. Carbachol dose response curves plotted by Prism (GraphPad).
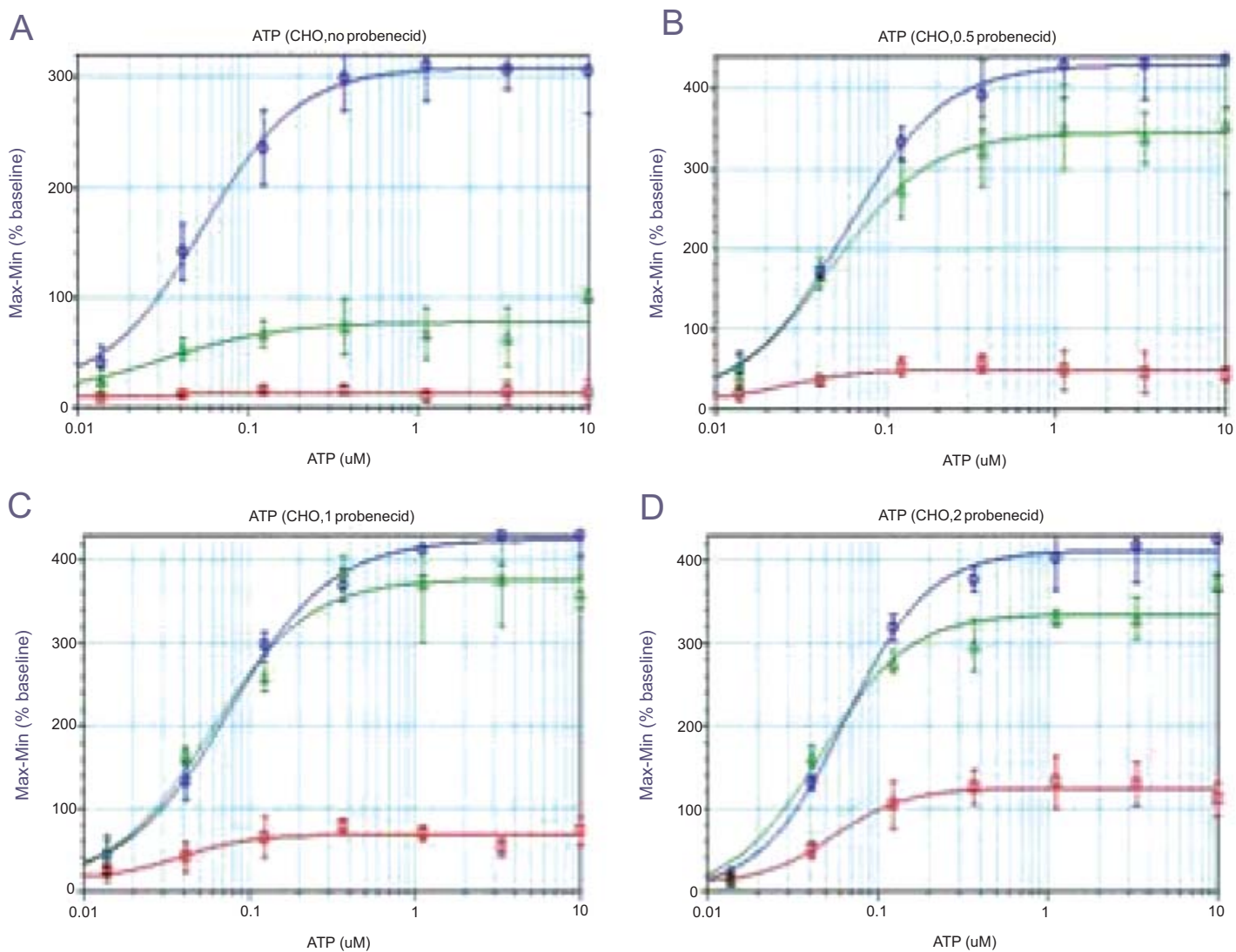

$\mathrm{BD}^{\mathrm{TM}} \mathrm{PBX}$ Culcium Assay Kit

$\mathrm{BD}^{\mathrm{TM}}$ Culcium Assay Kit

Culcium Kit

$\mathrm{n}=4$

Fig. (5). Probenecid titrations on CHO-M1 cells using different kits in 384-well format.

A. ATP dose response curves in the absence of probenecid.

B. ATP dose response curves in the presence of $0.5 \mathrm{mM}$ probenecid.

C. ATP dose response curves in the presence of $1 \mathrm{mM}$ probenecid.

D. ATP dose response curves in the presence of $2 \mathrm{mM}$ probenecid.

Recently, further improvements were developed that enhance the performance of the PBX calcium assay kit, $\left(\mathrm{BD}^{\mathrm{TM}}\right.$ PBX High Performance Calcium Assay Kit). Compared with the first generation kit, the new formulation provides higher

signal to background while maintaining EC50 values (Fig. 7). Both kits can be miniaturized to 1536-well format with high Z' values (Fig. 8). 


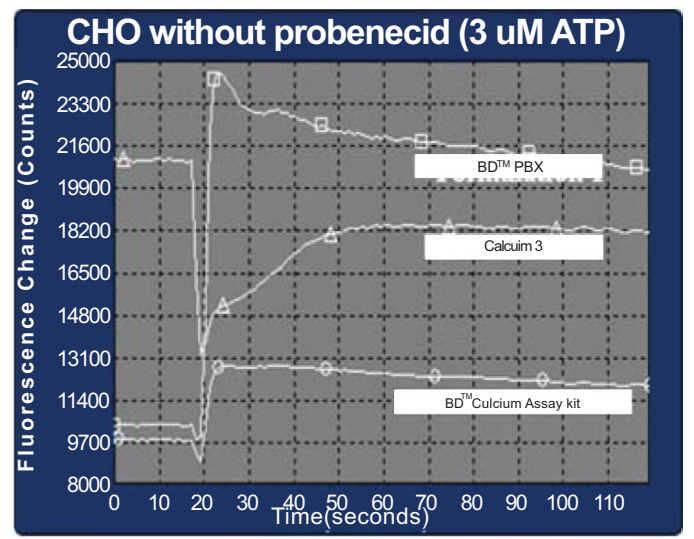

Fig. (6). Comparison of homogeneous calcium assays on FLIPR 3 (384-well).

Kinetic data of CHO-K1 cells treated with $3 \mu \mathrm{M}$ ATP.

$\square \mathrm{BD}^{\mathrm{TM}}$ PBX Calcium Assay Kit.

$\bigcirc \mathrm{BD}^{\mathrm{TM}}$ Calcium Assay Kit.

$\triangle$ Calcium 3 Kit.

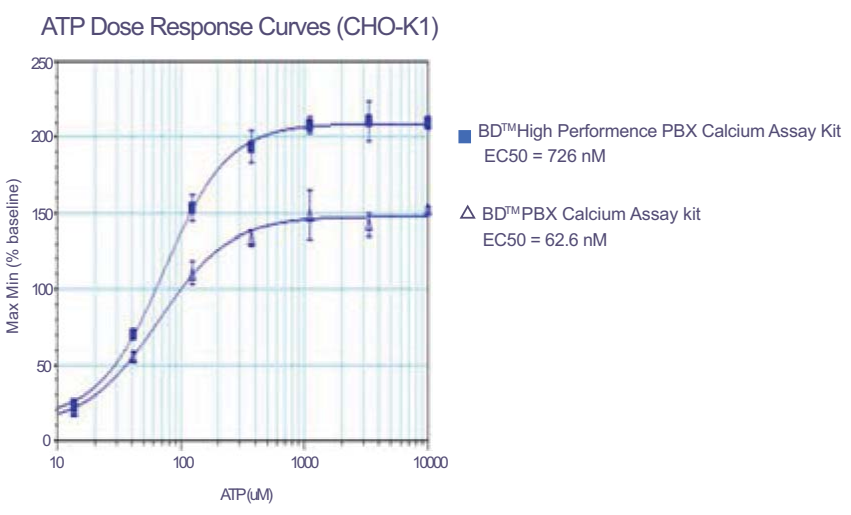

Fig. (7). Comparison of first and second generation $\mathbf{B D}^{\mathrm{TM}} \mathbf{P B X}$ calcium assay kit in 384-well format

ATP dose response curves of CHO-K1 cells in the absence of probenecid. Signal to background are 3.2 and 2.5, EC50 values are $62.6 \mathrm{nM}$ and $72.6 \mathrm{nM}$ using the first and second generation kits, respectively.

\section{DISCUSSION}

The BD calcium assay kit product family was tested against previous kit formulations and to other commercially available homogeneous calcium assay kits, and was found to provide better performance on the cell lines and receptors tested. The formulation of $\mathrm{BD}^{\mathrm{TM}}$ Calcium Assay Kits includes a unique signal-enhancing reagent to maximize the signal over background. Components such as enhancers, quenchers and other molecules can cause interference with test compounds, particularly small peptides, resulting in potential false negatives. During development of the current formulations, we tested a diversity of cell lines (e.g. CHOK1, HEK293, U2OS, HeLa) and receptors (e.g. A2b receptor, $\beta$-adrenoceptor, DRD5 receptor, CRF2 receptor, Glucagon receptor, PACAP receptor, secretin receptor, EP4 receptor, GIP receptor, muscarinic receptor, P2Y receptor, hista-
A
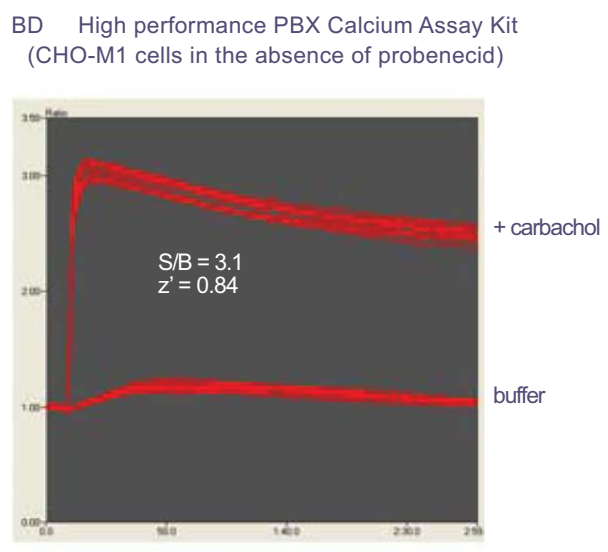

B

BD PBX Calcium Assay Kit (CHO-M1 cells in the absence of probenecid)

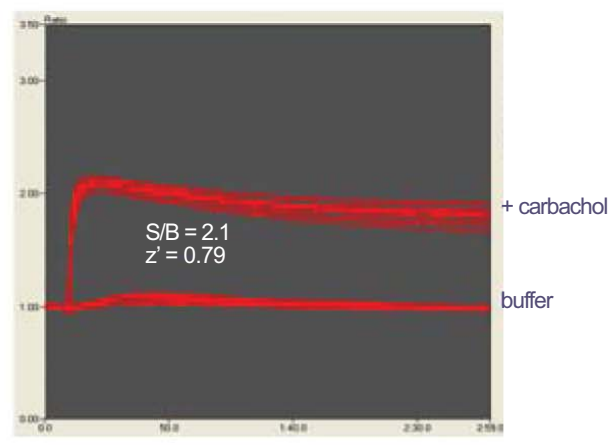

Fig. (8). Comparison of first and second generation $\mathrm{BD}^{\mathrm{TM}} \mathrm{PBX}$ calcium assay kit in 1536-well format

A. CHO-M1 cells treated with and without $1 \mu \mathrm{M}$ carbacol using $\mathrm{BD}^{\mathrm{TM}}$ High Performance PBX Calcium Assay Kit in absence of probenecid. Signal to background is 3.1 , and $Z$ ' value is $0.84(\mathrm{n}=$ 12).

B. CHO-M1 cells treated with and without $1 \mu \mathrm{M}$ carbachol using $\mathrm{BD}^{\mathrm{TM}}$ PBX Calcium Assay Kit in absence of probenecid. Signal to background is 2.1 , and Z' value is 0.79 .

mine $\mathrm{H} 1$ receptor, $\mathrm{CCR} 2$ receptor and $\mathrm{MCH}$ receptor 2) in an attempt to exclude interfering components. Following commercialization, others have extensively validated these formulations on their own well-characterized assays (personal communication). To our knowledge, no adverse pharmacological effects of the formulations' components on receptors or cells have been reported. However, because biological events are complicated and each cell line and receptor behaves differently, it is often valuable to evaluate several calcium assay formulations to identify the best performer for the specific assay and target.

In conclusion, we have developed a second generation BD calcium assay kits that provide higher signal to background and better performance than the previous generation kits. The new kits utilize novel fluorescent calcium indicators with improved dye-loading and retention efficiency. The 
improvements have enabled better data quality on calcium

[6] Visegrády A, Boros A, Némethy Z, Kiss B, Keseru GM. Application of the BD ACTOne ${ }^{\mathrm{TM}}$ Technology for the High-Throughput Screening of $\mathrm{G}_{\mathrm{s}}$-Coupled Receptor Antagonists. J Biomol Screen 2007; 12: 1068-1073.

ment.

\section{REFERENCES}

[7] Liu K, Titus S, Southall N, et al. Comparison of Functional Assays for Gq-coupled GPCRs by Measuring Inositol Monophosphate and Intracellular Calcium in 1536-well Plate Format. Current Chemical

[1] Hopkins AL, Groom CR. The druggable genome. Nat Rev Drug Discov 2002; 1(9): 727-30.

[2] Coward P, Chan SD, Wada HG, Humphries GM, Conklin BR. Chimeric $\mathrm{G}$ proteins allow a high-throughput signaling assay of Gicoupled receptors. Anal Biochem 1999; 270: 242-8.

[3] Zhang Y, Kowal D, Kramer A, Dunlop J. Evaluation of FLIPR Calcium 3 Assay Kit-a new no-wash fluorescence calcium indicator reagent. J Biomol Screen 2003; 8: 571-77.

[4] Xin H, Wang Y, Todd MJ, Qi J, Minor LK. Evaluation of no-wash calcium assay kits: enabling tools for calcium mobilization. J Biomol Screen 2007; 12: 705-14.

[5] Di Virgilio F, Steinberg TH, Silverstein SC. Inhibition of Fura-2 sequestration and secretion with organic anion transport blockers. Genomics 2008; in press.

[8] Werry TD, Christie MI, Dainty IA, Wilkinson GF, Willars GB $\mathrm{Ca}^{2+}$ signalling by recombinant human CXCR2 chemokine receptors is potentiated by $\mathrm{P} 2 \mathrm{Y}$ nucleotide receptors in HEK cells. Br J Pharmacol 2002; 135: 1199-1208.

[9] Iredale PA, Hill SJ. Increases in intracellular calcium via activation of an endogenous P2-purinoceptor in cultured CHO-K1 cells. Br J Pharmacol 1993; 110: 1305-11.

[10] Zhang JH, Chung TD, Oldenburg KR. A simple statistical parameter for use in evaluation and validation of high throughput screening assays. J Biomol Screen 1999; 4: 67-73. Cell Calcium 1990; 11: 57-62.

(C) Li et al.; Licensee Bentham Open.

This is an open access article licensed under the terms of the Creative Commons Attribution Non-Commercial License (http://creativecommons.org/licenses/by-nc/3.0/) which permits unrestricted, non-commercial use, distribution and reproduction in any medium, provided the work is properly cited. 